\title{
Article \\ Enhanced Seroconversion to West Nile Virus Proteins in Mice by West Nile Kunjin Replicon Virus-like Particles Expressing Glycoproteins from Crimean-Congo Hemorrhagic Fever Virus
}

\author{
Pham-Tue-Hung Tran ${ }^{1}$ (D), Urban Höglund ${ }^{2}$, Olivia Larsson ${ }^{2}$, Sofia Appelberg ${ }^{3} \mathbb{D}$, Ali Mirazimi ${ }^{4,5}$, \\ Magnus Johansson ${ }^{1}$ and Wessam Melik ${ }^{1, *(D)}$
}

Citation: Tran, P.-T.-H.; Höglund, U.; Larsson, O.; Appelberg, S.; Mirazimi,

A.; Johansson, M.; Melik, W.

Enhanced Seroconversion to West Nile Virus Proteins in Mice by West Nile Kunjin Replicon Virus-like Particles Expressing Glycoproteins from Crimean-Congo Hemorrhagic Fever Virus. Pathogens 2022, 11, 233 https://doi.org/10.3390/ pathogens 11020233

Academic Editor: Enrico Lavezzo

Received: 12 January 2022

Accepted: 7 February 2022

Published: 10 February 2022

Publisher's Note: MDPI stays neutral with regard to jurisdictional claims in published maps and institutional affiliations.

Copyright: (C) 2022 by the authors. Licensee MDPI, Basel, Switzerland. This article is an open access article distributed under the terms and conditions of the Creative Commons Attribution (CC BY) license (https:// creativecommons.org/licenses/by/ $4.0 /)$
1 School of Medical Science, Inflammatory Response and Infection Susceptibility Centre (iRiSC), Örebro University, 70362 Örebro, Sweden; hung.tran@oru.se (P.-T.-H.T.); magnus.johansson@oru.se (M.J.)

2 Adlego Biomedical AB, P.O. Box 42, 75103 Uppsala, Sweden; urban.hoglund@adlego.se (U.H.); olivia.larsson@adlego.se (O.L.)

3 Department of Microbiology, Public Health Agency of Sweden, 17182 Solna, Sweden; sofia.appelberg@folkhalsomyndigheten.se

4 Division of Clinical Microbiology, Department of Laboratory Medicine, Karolinska Institution, 14152 Huddinge, Sweden; ali.mirazimi@ki.se

5 National Veterinary Institute, 75189 Uppsala, Sweden

* Correspondence: wessam.melik@oru.se

\begin{abstract}
Removal of genes coding for major parts of capsid (C), premembrane (prM), and envelope (E) proteins on the flavivirus genome aborts the production of infectious virus particles where the remaining genome forms a replicon that retains replicability in host cells. The C-prM-E proteins can also be expressed in trans with the flavivirus replicons to generate single-round infectious replicon virus-like particles (RVPs). In this study, we characterized the use of RVPs based on the Kunjin strain of $\mathrm{WNV}\left(\mathrm{WNV}_{\mathrm{KUN}}\right)$ as a putative $\mathrm{WNV}$ vaccine candidate. In addition, the $\mathrm{WNV}_{\mathrm{KUN}} \mathrm{C}$-prM-E genes were substituted with the Crimean-Congo hemorrhagic fever virus (CCHFV) genes encoding the glycoproteins $\mathrm{Gn}$ and $\mathrm{Gc}$ to generate a $\mathrm{WNV}_{\mathrm{KUN}}$ replicon expressing the CCHFV proteins. To generate RVPs, the $\mathrm{WNV}_{\mathrm{KUN}}$ replicon was transfected into a cell line expressing the $\mathrm{WNV}_{\mathrm{KUN}} \mathrm{C}$-prM-E. Using immunoblotting and immunofluorescence assays, we showed that the replicon can express the CCHFV Gn and Gc proteins and the RVPs can transduce cells to express $\mathrm{WNV}_{\mathrm{KUN}}$ proteins and the CCHFV Gn and Gc proteins. Our study also revealed that these RVPs have potential as a vaccine platform with low risk of recombination as it infects cells only in one cycle. The immunization of mice with the RVPs resulted in high seroconversion to both WNV E and NS1 but limited seroconversion to CCHFV Gn and Gc proteins. Interestingly, we found that there was enhanced production of WNV E, NS1 antibodies, and neutralizing antibodies by the inclusion of CCHFV Gc and Gn into WNV $\mathrm{KUN}_{\mathrm{N}}$ RVPs. Thus, this study indicates a complementary effect of the CCHFV Gn and Gc proteins on the immunogenicity by $\mathrm{WNV}_{\mathrm{KUN}}$ RVPs, which may be applied to develop a future vaccine against the WNV.
\end{abstract}

Keywords: replicon virus-like particles (RVPs); replicons; West Nile Kunjin virus; envelope; nonstructural protein 1; Crimean-Congo hemorrhagic fever virus; glycoprotein (Gn-Gc); seroconversion; neutralization; vaccines

\section{Introduction}

The neurotropic West Nile virus (WNV) consists of many lineages, among which the lineage 1 has been associated with the majority of outbreaks [1]. In this group, infections of the New York 1999 strain $\left(\mathrm{WNV}_{\mathrm{NY}}\right.$ ) $)$ can result in severe meningoencephalitis with a fatality rate around 1\% [2]. However, there is no approved human vaccine or antiviral treatment for the infection. In the same lineage, infections of the Kunjin strain $\left(\mathrm{WNV}_{\mathrm{KUN}}\right)$, a naturally attenuated WNV, has resulted in only a few nonfatal disease cases since $1960[3,4]$. 
Furthermore, $\mathrm{WNV}_{\mathrm{NY} 99}$ and $\mathrm{WNV}_{\mathrm{KUN}}$ share $89 \%$ of the envelop (E) gene, $88 \%$ of the non-structure (NS) 5 gene and the $3^{\prime}$ untranslated region (UTR) [3]. Therefore, $\mathrm{WNV}_{\mathrm{KUN}}$ could be used as a strategy to develop a vaccine against $\mathrm{WNV}_{\mathrm{NY} 99}$.

$\mathrm{WNV}_{\mathrm{NY} 99}$ and $\mathrm{WNV}_{\mathrm{KUN}}$ are members of the family Flaviviridae, which are enveloped viruses with icosahedral structures. The viral genome, which is an approximately $11 \mathrm{~kb}$ positive-sense single-stranded RNA (+ssRNA), codes for a single polyprotein. Cleavages of the polyproteins by viral and cellular proteases result in three structural proteinscapsid (C), precursor membrane (prM), and E for viral assembly, as well as seven NS proteins. In addition, the viral genomic RNA is flanked by $5^{\prime}$ - and $3^{\prime}$-UTRs which are required for translation, replication, and assembly [5-8].

The removal of the C-prM-E genes in the flavivirus genome results in replicons that can replicate in transfected cells but abolish production of infectious virus particles [9-12]. $\mathrm{WNV}_{\mathrm{KUN}}$ was one of the first flavivirus replicon systems constructed [13]. The replicon can be co-expressed with the corresponding structural genes, resulting in the packaging of replicon and the generation of replicon virus-like particles (RVPs). Several studies have demonstrated the potential of flavivirus RVPs as a vaccine platform [14-17].

Crimean-Congo hemorrhagic fever virus (CCHFV) is a highly virulent virus that has a fatality rate between 5 and $30 \%$, depending on the outbreaks [18]. As with other members of the genus Orthonairovirus and family Nairoviridae, the virus is an enveloped negative-sense RNA virus. The virus has three genomic segments: small, medium, and large encoding nucleoproteins; glycoproteins; and RNA-dependent RNA polymerase, respectively. The virus infects humans mainly through Hyalomma ticks, which live throughout Africa, Southern and Eastern Europe, the Middle East, India, and Asia [19].

As flavivirus RVPs can be used as a vector to transduce cells and express proteins of interest, they are potentially employed as a multiple vaccine platform. Though CCHFV and WNV infect human and livestock by different vectors, i.e., tick and mosquito, respectively, there are overlapping geographic distributions of the two viruses in Western Asia and Balkan Europe [20-23]. In this study, we generated $\mathrm{WNV}_{\mathrm{KUN}} \mathrm{RVPs}$ that deliver genes coding glycoproteins CCHFV Gn and Gc to infected cells. These RVPs were then administered to mice to examine their potential as a vaccine candidate. The administration of RVPs into mice induced seroconversion, generating antibodies against CCHFV Gn, CCHFV Gc, $\mathrm{WNV}_{\mathrm{NY} 99} \mathrm{NS1}$, and WNV $\mathrm{NY}_{\mathrm{N} 9} \mathrm{E}$. However, serum from the CCHFV Gn-Gc RVP-injected mice limitedly neutralize CCHFV but enhanced the neutralization of $\mathrm{WNV}_{\mathrm{KUN}}$ and seroconversion to $\mathrm{WNV}_{\mathrm{NY} 99} \mathrm{NS1}$ and $\mathrm{E}$. The data in this study highlight a strategy using the $\mathrm{WNV}_{\mathrm{KUN}}$ RVPs with the CCHFV Gn-Gc as a vaccine against WNV.

\section{Results}

\subsection{CCHFV Gn-Gc Expression by the $W N V_{K U N}$ Replicon}

As we aimed to generate mutivalent $\mathrm{WNV}_{\mathrm{KUN}}$ RVPs that can transduce the CCHFV Gn-Gc gene, we initially generated a DNA $W_{N V_{K U N}}$ replicon that can express the CCHFV glycoproteins $\mathrm{Gn}$ and Gc, termed Gn-Gc replicon. Here, the luciferase (Luc) reporter gene from the previously described $\mathrm{WNV}_{\mathrm{KUN}}$ replicon [24] was substituted with genes encoding CCHFV Gn-Gc with the foot-and-mouth disease virus autoprotease 2a (FMDV2A) gene inserted between Gn and Gc, allowing for the cleavage of the Gn-Gc into Gn and Gc during replicon expression (Figure 1A). The replicons were then transfected into BHK-21 cells stably expressing $\mathrm{WNV}_{\mathrm{KUN}} \mathrm{C}-\mathrm{prM}-\mathrm{E}$, as described previously [24]. Compared to the Luc replicon, the Gn-Gc replicon expressed the CCHFV Gn and Gc protein as expected (Figure 1B). In addition, both replicons also expressed the $\mathrm{WNV}_{\mathrm{KUN}} \mathrm{NS1}$ protein (Figure 1B). All together, these suggest the replicons can express the $\mathrm{WNV}_{\mathrm{KUN}}$ polyprotein and the inserted CCHFV Gn and Gc genes. 
A

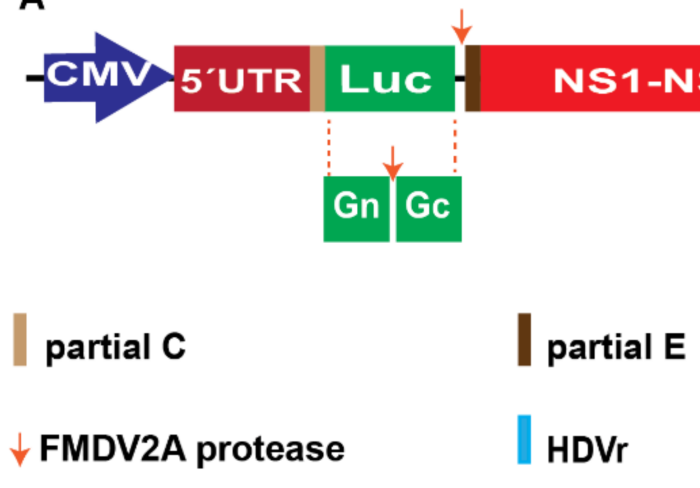

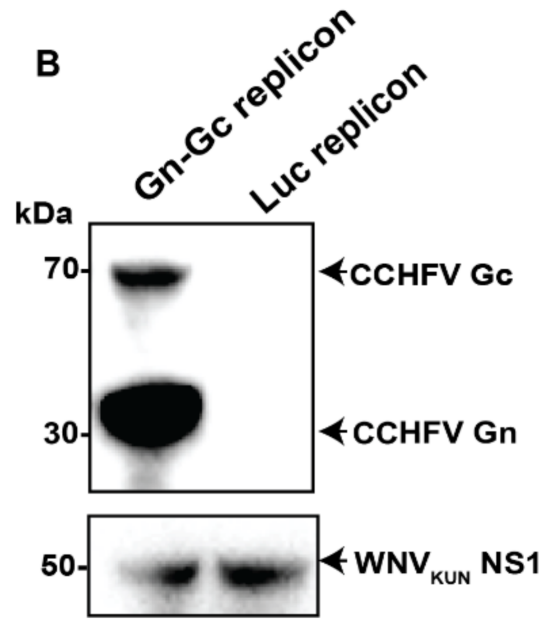

Figure 1. Expression of Crimean-Congo hemorrhagic fever virus (CCHFV) glycoproteins Gn and GC proteins using the West Nile Kunjin $\left(\mathrm{WNV}_{\mathrm{KUN}}\right)$ replicon. (A) The reporter luciferase (Luc) gene was substituted with genes encoding CCHFV Gn and Gc in the $W_{N V} V_{K N N}$ DNA replicon. In short, the replicon is driven by the cytomegalovirus (CMV) promoter expressing an open reading frame flanked by the $5^{\prime}$ - untranslated region (UTR) and the $3^{\prime}$-UTR comprising: first, partial capsid (C) gene fused in frame with the Luc, the foot and mouth disease virus autoprotease 2a (FMDV2A) then partial envelop (E) gene, and all the nonstructural proteins. The hepatitis delta virus antigenomic ribozyme (HDVr) sequence was inserted immediately downstream of the $\mathrm{WNV}_{\mathrm{KUN}} 3^{\prime}$-UTR followed by the Simian virus 40 (SV40) polyadenylation signal (pA). (B) Immunoblotting of cell lysates 2 days after transfection of the $\mathrm{WNV}_{\mathrm{KUN}} \mathrm{CCHFV} \mathrm{Gn}-\mathrm{Gc}$ replicons versus the $\mathrm{WNV}_{\mathrm{KUN}}$ Luc replicon into the BHK-21 cell line expressing the $\mathrm{WNV}_{\mathrm{KUN}} \mathrm{C}$-prM-E.

\section{2. $W N V_{K U N} R V P s$ Transduced Their Polyprotein Gene and CCHFV Gn-Gc Gene in A Single Cycle}

As the Gn-Gc replicon could express the Gn and the Gc proteins, we characterized the packaging of the replicon by the C-prM-E proteins to generate RVPs and its safety. We initially transfected the $\mathrm{Gn}-\mathrm{Gc}$ replicon into the $\mathrm{WNV}_{\mathrm{KUN}} \mathrm{C}$-prM-E BHK-21 cell line as previously described [24]. Here, CCHFV Gn, Gc, and WNV KUN NS1 could be visualized, indicating replicon expression, and staining of dsRNA revealed replicon replication (Figure 2).

As the cell line can express the C-prM-E proteins, the replicon can be encapsidated and enveloped to generate RVPs. To characterize this postulation, we collected the supernatant from transfected cells and infected naïve BHK-21 cells in two consecutive cycles. Media from the RVP-generating cells were diluted to the dilutions with the highest numbers of cells infected. In the first round of infection, cells were transduced to express the CCHFV Gn, Gc, and WNV $\mathrm{KUN}_{\mathrm{N}}$ NS1 proteins, and immunostainings revealed replicon replication (Figure 2). Because naïve BHK-21 cells do not have the gene encoding C-prM-E, the produced replicon should not be able to be packaged to form new RVPs. As expected, BHK-21 infected in the second cycle could not express the replicon. These results indicate that the Gn-Gc replicon can be packaged to generate RVPs and that the RVPs infect cells only in a single cycle, similarly to the Luc RVPs [24]. 


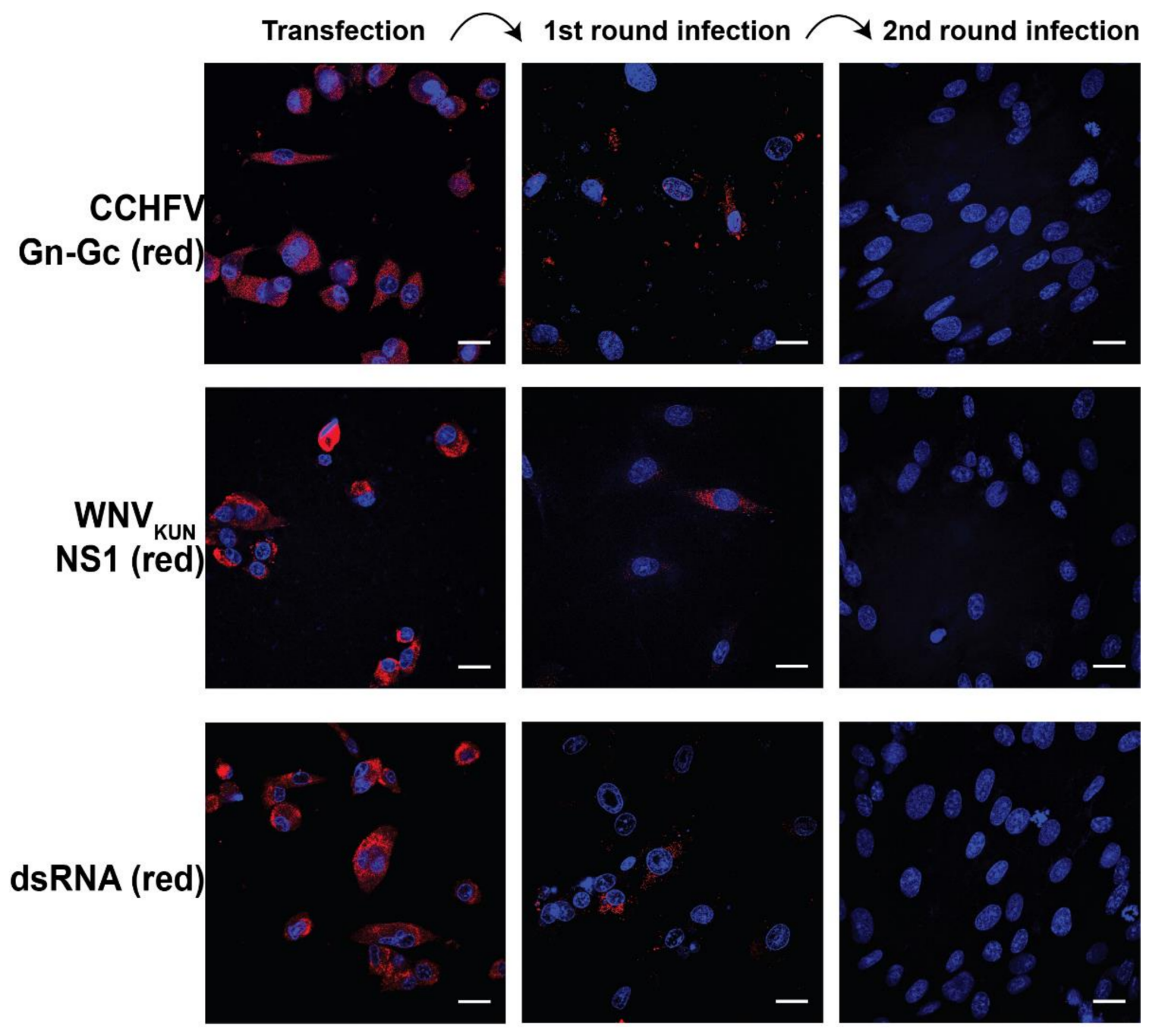

Figure 2. Immunofluorescence labeling of BHK-21 C-prM-E cells after transfection with the Gn-Gc replicon, followed by two consecutive cycles of RVP infection into naïve BHK-21 cells. The cells were visualized with the antibodies anti-CCHFV Gn-Gc (red), anti-WNV $\mathrm{KUN}_{\mathrm{N}} \mathrm{NS1}$ (red), and anti-dsRNA (red). The nucleus was counterstained with DAPI (blue). Bar scales represent $20 \mu \mathrm{m}$.

2.3. CCHFV Gn-Gc RVPs Induced Seroconversion to CCHFV Gn and Gc Proteins and Enhanced Seroconversion to WNV $V_{N Y 99}$ NS1 and E Proteins

As we demonstrated that RVPs could transduce cells in vitro, we examined the in vivo immunogenicity by administering them into mice. Mice were divided into three groups and subcutaneously injected with either Gn-Gc RVPs, Luc RVPs, or PBS (Figure 3A). During the study, mice from all groups were healthy and showed no significant differences in weight (Figure 3B).

We then determined the antibody titers in sera. Mice immunized with CCHFV Gn-Gc RVPs showed seroconversions to $\mathrm{Gn}$ and Gc proteins with the serum titers reaching 1:250 and 1:50 dilutions, respectively (Figure 3C,D). As there were seroconversions to Gn and Gc proteins by mice injected with the control Luc RVPs, there might be cross-reactivity of $\mathrm{WNV}_{\mathrm{KUN}}$ RVPs antibodies to the Gn-Gc protein. 
A
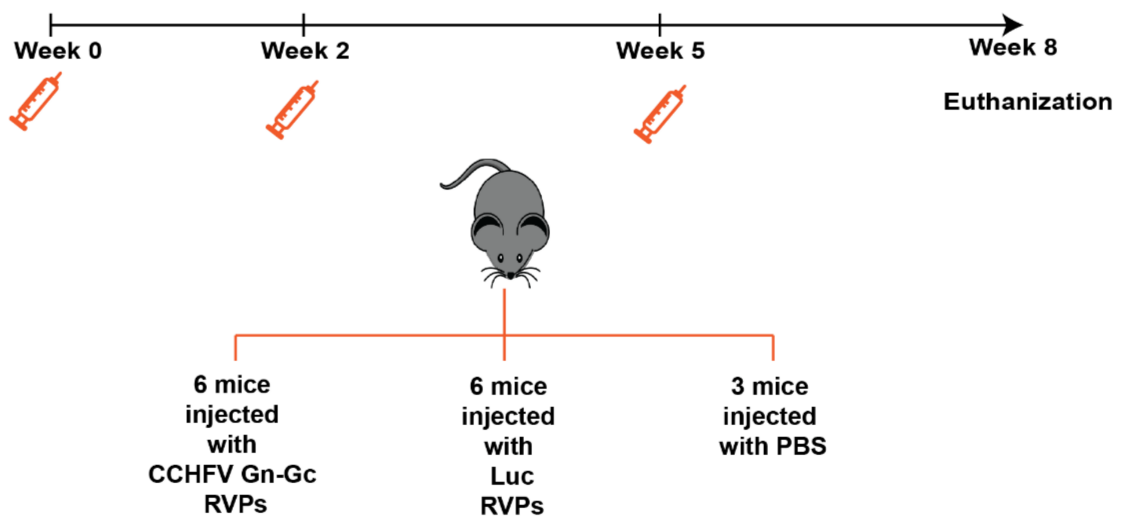

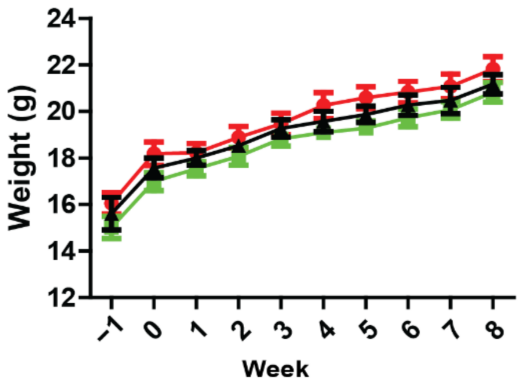

$\rightarrow$ CCHFV Gn-Gc

$\mp$ PBS

E WNV $_{\text {NY99 }} E$

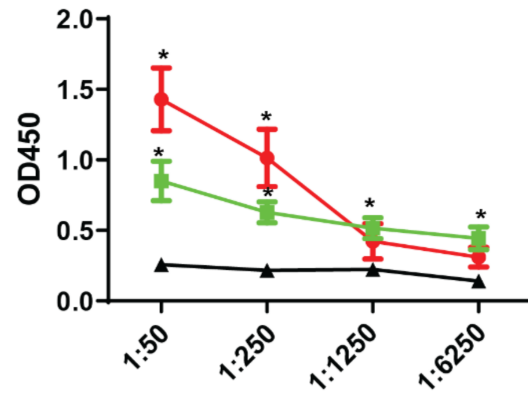

Serum dilutions
C

CCHFV Gn

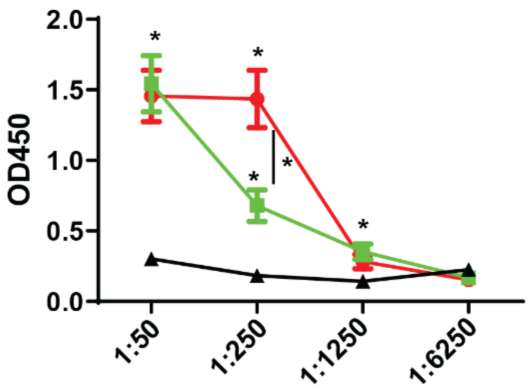

Serum dilutions

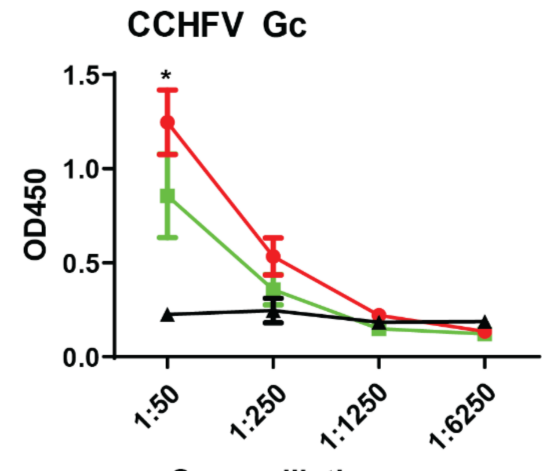

Serum dilutions

G WNV $_{\text {KUN }}$ neutralization

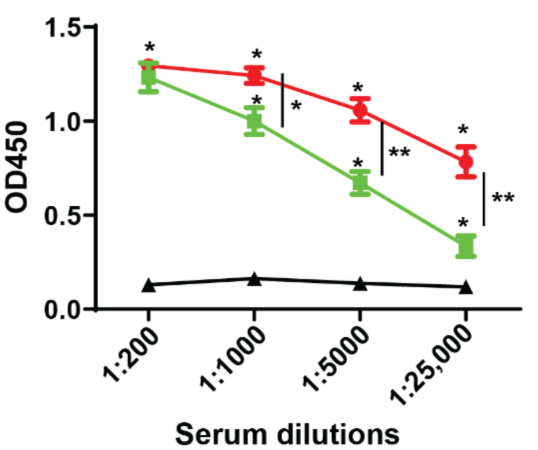

Figure 3. CCHFV Gn-Gc RVPs induced seroconversion to CCHFV Gn and Gc and enhanced seroconversion to $\mathrm{WNV}_{\mathrm{NY} 99} \mathrm{NS1}$, E. (A) Schematic illustration of the mice immunization schedule. Mice were subcutaneously immunized three times at weeks 0,2 , and 5 with RVPs expressing CCHFV Gn-Gc (6 mice), Luc (6 mice), or phosphate-buffered saline (PBS) (3 mice). (B) Mouse weight from one week before the experiment to the mouse-euthanized day. Mice sera from the study groups were diluted and assayed with enzyme-linked immunosorbent assays (ELISA) to measure antibody

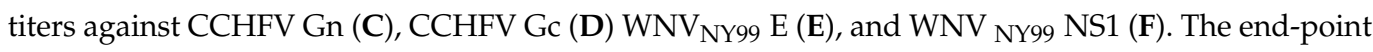
titers were determined as there was no difference in the measured optical density values at $450 \mathrm{~nm}$ (OD450) between the vaccinated group and the control group. The experiments were conducted with two technical repeats. The $\mathrm{p}$ values are indicated using ${ }^{*} p<0.05$ and ${ }^{* *} p<0.01$. (G) Serum titers that elicited $50 \%$ reduction in the $\mathrm{WNV}_{\mathrm{KUN}}$ plaque number. Sera from experimented animal were combined before assaying. 
As the $\mathrm{WNV}_{\mathrm{KUN}}$ replicon is packaged by C-prM-E to generate RVPs and the $\mathrm{WNV}_{\mathrm{KUN}}$ is the attenuated strain of $\mathrm{WNV}_{\mathrm{NY} 99}[3,4]$, we determined the seroconversion of the RVPs to the $\mathrm{WNV}_{\mathrm{NY} 99} \mathrm{E}$ and NS1. Mice immunized with either Gn-Gc RVPs or Luc RVPs showed seroconversion to the $\mathrm{WNV}_{\mathrm{NY} 99} \mathrm{E}$ and NS1, whose antibody titers can reach to more than 1:6250 and 1:25,000 dilutions, respectively (Figure 3E,F). Interestingly, there was a significant enhancement of seroconversion to these proteins in mice immunized by the Gn-Gc RVPs, compared to the Luc RVPs.

Ultimately, we examined virus neutralization by sera. As $\mathrm{WNV}_{\mathrm{KUN}}$ share $89 \%$ of the envelop (E) gene to $\mathrm{WNV}_{\mathrm{NY} 99}[3]$ and the $\mathrm{WNV}_{\mathrm{KUN}}$ virus in a neutralization assay is often employed in the context of $\mathrm{WNV}_{\mathrm{NY} 99}$ vaccine development $[25,26]$, neutralization to $\mathrm{WNV}_{\mathrm{KUN}}$ by the sera was examined. As expected, pooled sera from all mice administrated with the Gn-Gc RVPs showed the enhanced effect of neutralizing the $\mathrm{WNV}_{\mathrm{KUN}}$, as the antibody titer with a 50\% reduction in plaque numbers was 1:250, compared to 1:50 in mice administered with Luc RVPs (Figure 3G).

To eliminate the effect of cross-reactivity, we examined CCHFV neutralization by the sera from the Gn-Gc RVP group compared to the Luc RVP group. Sera from the Gn-Gc RVPs were divided into two groups based on a high or low level of antibodies before assaying. In accordance with the antibody levels of the two groups, at the first serum dilution (1:8), diluted sera from the mice with a higher Gc antibody level and these from the mice with a lower Gc antibody level showed around 35\% and 15\% neutralization of infection, respectively, compared to sera from the control (RVPs-Luc) immunized mice (data not shown).

These data suggest that the RVPs can induce seroconversion to antibodies against the CCHFV and $\mathrm{WNV}_{\mathrm{NY} 99}$ proteins. In addition, the CCHFV Gn-Gc can enhance the seroconversion to the $\mathrm{WNV}_{\mathrm{NY} 99}$ proteins, suggesting that they can be incorporated to the $W_{\text {KUN }}$ RVPs, enhancing the potentials of the RVPs as a putative vaccine candidate against WNV.

\section{Discussion}

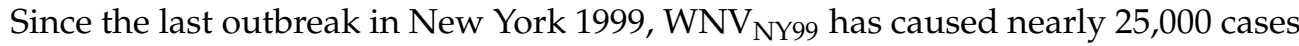
with neuronal invasive symptoms (encephalitis and meningitis) and more than 2000 deaths in North America [2]. In Europe, the virus has caused more than 2000 disease cases and 181 deaths [27]. Thus, there is need to have approved vaccines against the virus, preventing future pandemic burden. There have been six vaccine candidates against WNV in the clinical trial phases I and II, including DNA vector expressing $\mathrm{WNV}_{\mathrm{NY} 99} \mathrm{E}$, recombinant $\mathrm{WNV}_{\mathrm{NY} 99}$ E protein, inactivated $\mathrm{WNV}_{\mathrm{NY} 99}$, inactivated $\mathrm{WNV}_{\mathrm{KUN}}$, live chimeric $\mathrm{WNV}_{\mathrm{NY} 99}{ }^{-}$ dengue 4 virus, and live chimeric $W_{N V_{N Y 99}-y e l l o w}$ fever virus $17 \mathrm{D}[28,29]$.

As $\mathrm{WNV}_{\mathrm{NY} 99}$ neurological disease is more severe in the elderly, the major target of vaccines against the virus should be the immunosenescent population. In this context, the immunogenicity and safety of the vaccine are essential. The $\mathrm{WNV}_{\mathrm{KUN}} \mathrm{RVP}$ platform is based on the $\mathrm{WNV}_{\mathrm{KUN}}$ virus, which is an attenuated strain of WNV [3,4]. As the particle contains replicons with no viral packaging genes, it can infect cells in a single cycle, abolishing systemic infection. As a strategy for vaccine development, it is a compromise between inactivated and live-attenuated virus vaccines. Unlike inactivated virus particles, $W_{N V_{K U N}}$ RVPs can preferentially infect and transduce antigen-presenting cells, such as macrophages or dendritic cells [30-33], and express vaccine candidate proteins, therefore inducing a greater immune response. Compared to live-attenuated virus vaccines, the infectivity of RVPs is limited to a single round, which makes them a safer vaccine platform for the immunosenescent population.

In this study, we characterized the expression and the safety of $\mathrm{WNV}_{\mathrm{KUN}}$ RVPs, which deliver CCHFV Gn and Gc. Without the CCHFV nucleoprotein, these two proteins cannot function as an CCHFV packaging system. Thus, there is no risk for generation of a chimeric $\mathrm{WNV}_{\mathrm{KUN}}-\mathrm{CCHFV}$ virus using the RVP platform. Indeed, we showed that the RVPs with these genes only infected cells in a single round and mice injected with the RVP survived. 
Immunization of the RVPs elicited seroconversions to the antibodies in mice. The GnGc RVPs also conferred the production of antibodies against the $\mathrm{WNV}_{\mathrm{NY} 99} \mathrm{NS1}$ and E, given that $\mathrm{WNV}_{\mathrm{KUN}}$ is an attenuated strain of $\mathrm{WNV}_{\mathrm{NY} 99}$. Interestingly, the expression of CCHFV Gn and Gc enhanced the immunogenicity of the $\mathrm{WNV}_{\mathrm{NY} 99} \mathrm{E}, \mathrm{NS1}$, and $\mathrm{WNV}_{\mathrm{KUN}}$ neutralization, which requires further investigation. Indeed, many epitopes within the $\mathrm{CCHFV} \mathrm{Gn}$ and Gc have been predicted to induce an immune response to $\mathrm{CD} 8^{+}, \mathrm{CD}^{+}$, and linear $\mathrm{B}$ cells, which may suggest the enhanced antibody production to $\mathrm{WNV}_{\mathrm{NY} 99} \mathrm{E}$ and NS1 by Gn and Gc [34,35].

Furthermore, the Gn-Gc RVPs from this study could not induce strong neutralizing antibodies against CCHFV. Likewise, the neutralizing antibody response from CCHFV survivors is also usually low, ranging from 1:8 to 1:32 according to fluorescent focus reduction tests [36]. Interestingly, epitope mapping from CCHFV survivors indicated that antibody responses toward epitopes within Gn and Gc are not likely to elicit neutralizing antibodies [37], but passive immunization with these antibodies was able to protect against CCHFV, highlighting the roles of other mechanisms, such as antibody-dependent cell-mediated cytotoxicity. Thus, although mice sera did not strongly induce CCHFV neutralization, future studies should examine the T cell response to the CCHFV by the Gn-Gc RVPs.

\section{Materials and Methods}

\subsection{Cell Culture}

Baby hamster kidney (BHK-21) (ATCC) and Vero (ATCC) cells were maintained in Dulbecco's modified Eagle's medium (DMEM) containing $1 \mathrm{~g} / \mathrm{L}$ of glucose (Gibco, Paisley, UK), supplemented with $10 \%$ heat-inactivated fetal bovine serum (HI-FBS) (Gibco) and $100 \mathrm{U} / \mathrm{mL}$ of penicillin-streptomycin (PEST) (Gibco) at $37^{\circ} \mathrm{C}$ in $5 \% \mathrm{CO}_{2}$.

\subsection{Preparation of Gene Constructs}

The $W N V_{K U N}$ replicon was constructed based on the $W N V_{K U N}$ sequence (accession number AY274504), as described previously [24]. To generate the $\mathrm{WNV}_{\mathrm{KUN}}$ replicon expressing the CCHFV Gn and Gc, the CCHFV Hoti strain Gn and Gc genes were PCR-amplified from the M segment cDNA clone (accession number MH483985.1) with suitable primers to create a FMDV2A between the Gn and Gc and suitable restriction sites flanking the Gn-Gc. The fragment was then substituted with the Luc gene in the $\mathrm{WNV}_{\mathrm{KUN}}$ replicon by suitable restriction reactions and ligations.

\subsection{Proteins and Antibodies}

The following proteins and antibodies were used in this study: the $W_{N V} V_{N Y 99} E$ protein, the $\mathrm{WNV}_{\mathrm{NY} 99} \mathrm{NS1}$ protein, the CCHFV Gn protein, the CCHFV Gc protein human Fc tag, the mouse anti-CCHFV Gn (The Native Antigen Company, Oxford, UK), the mouse anti-flavivirus NS1 (Abcam, Cambridge, UK), the mouse J2 anti dsRNA (Scicons, Szirák, Hungary), the mouse anti-CCHFV Gn-Gc (Friedrich-Loeffler-Institute, Greifswald, Germany), the Alexa Fluor 594-conjugated anti-mouse goat antibody (Invitrogen, Vilnius, Lithuania), and the HPR-conjugated anti-mouse goat antibody (Invitrogen).

\subsection{Protein Electrophoresis and Immunoblotting}

Protein electrophoresis and immunoblotting were conducted as previously described [24,38]. RIPA buffer (Thermoscientific, Waltham, MA, USA) with protease inhibitors (Sigma, St. Louis, MO, USA) was used to lyze cells for $20 \mathrm{~min}$ at $4{ }^{\circ} \mathrm{C}$, followed by boiling in LDS sample buffer (Invitrogen). Proteins were separated for $70 \mathrm{~min}$ at $120 \mathrm{~V}$ constant on precast $4-12 \%$ polyacrylamide Bis-Tris gels in MES running buffer (Invitrogen), followed by transfer to nitrocellulose membranes using the iBlot 2 Gel Transfer Device (Invitrogen). Proteins of interest were detected with the antibodies anti-CCHF Gn (1:1000), anti-CCHFV Gn-Gc (1:100), and anti-flavivirus NS1 (1:100). 


\subsection{Immunofluorescence Assay}

Cells were fixed by 4\% paraformaldehyde (Scharlau, Barcelona, Spain) for 20 min at room temperature (RT). They were then permeabilized by $0.1 \%$ Triton X-100 (VWR), followed by blocking with $2 \%$ bovine serum albumin (Fitzgerald, MA, USA) and 2\% goat serum (Invitrogen). Cells were labeled with the primary antibody anti-CCHFV Gn-Gc (1:100) for $2 \mathrm{~h}$ at RT, followed by an incubation with the secondary antibody Alexa Fluor 594 (1:500) for $1 \mathrm{~h}$ at RT. Images were captured using a confocal laser scanning microscopy SP8 (Leica, Wetzlar, Germany) and analyzed using ImageJ.

\subsection{RVP Purification and RVP Concentration Measurement}

The RVPs were purified as previously described [24]. In short, supernatants from the RVP production system were collected then loaded on $25 \%$ sucrose (Sigma), following by ultra-centrifugations. After centrifugation, both the supernatant and the sucrose were removed, and the RVPs were dissolved in DMEM. To monitor RVP concentration, diluted RVPs were utilized to infect confluent BHK-21 cells for $1 \mathrm{~h}$ at $37^{\circ} \mathrm{C}$ in $5 \% \mathrm{CO}_{2}$ incubator. The cells were immunofluorescent labeled two days after the infection to count the number of infected cells revealing the number of RVPs.

\subsection{Animal Immunizations}

Six-week-old female BALB/cN mice (Charles River, Freiburg, Germany) were administrated subcutaneously with $200 \mu \mathrm{L}$ of concentrated RVPs (approximately $10^{6}$ particles). Mice were divided into three groups and administrated: RVPs carrying replicons expressing CCHFV Gn-Gc (six mice), RVPs carrying replicons expressing Luc (six mice), or phosphate-buffered saline (PBS) (Gibco).

\subsection{Sample Collection}

Three weeks after the last RVP immunization, the mice were anesthetized with isoflurane for collection of retro-orbital blood samples, after which they were euthanized. The blood was incubated at RT for $30 \mathrm{~min}$ before centrifugation at $2000 \times \mathrm{g}$ for $10 \mathrm{~min}$ at $20{ }^{\circ} \mathrm{C}$. The sera were collected and stored at $-80{ }^{\circ} \mathrm{C}$ until use.

\subsection{Enzyme-Linked Immunosorbent Assays (ELISA)}

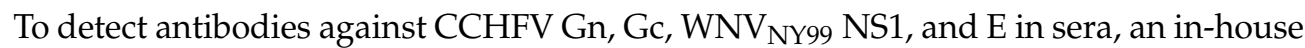
ELISA was developed using Nunc Maxisorb plates (Invitrogen) coated with the CCHFV

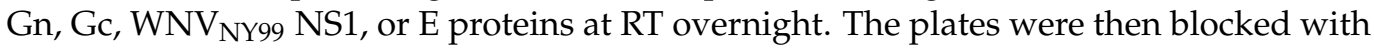
PBS with 1\% BSA for $2 \mathrm{~h}$ at RT and washed three times with PBST. Next, $100 \mu \mathrm{L}$ of diluted sera were added to each well and incubated for $1 \mathrm{~h}$ at RT on a shaker at $700 \mathrm{rpm}$. After washing, horseradish peroxidase (HRP)-conjugated anti-mouse antibody $(1: 10,000)$ was added and incubated at RT for $1 \mathrm{~h}$. After washing, $100 \mu \mathrm{L}$ of substrate solution (ABcam), containing tetramethylbenzidine and peroxide, was added. Plates were then incubated for $15 \mathrm{~min}$ before adding $100 \mu \mathrm{L}$ of stopping solution containing $1 \mathrm{M}$ of $\mathrm{HCl}$. The absorbance was read at $450 \mathrm{~nm}$ using the Cytation 3 Multi-Mode Reader (BioTek, Bad Friedrichshall, Germany).

\subsection{0. $W N V_{K U N}$ and CCHFV Neutralization Assays}

Sera collected from each group were pooled and heat-inactivated by incubation at $56{ }^{\circ} \mathrm{C}$ for $30 \mathrm{~min}$ before being 5-fold serially diluted in DMEM. WNV KUN was rescued from infectious clones, as previously described [38], and was diluted so that there were approximately 50 infectious particles per volume. Next, the diluted sera were incubated for $1 \mathrm{~h}$ at $37^{\circ} \mathrm{C}$ before infecting $90 \%$ confluent Vero cells growing on 24 well-plates for $1 \mathrm{~h}$ at $37{ }^{\circ} \mathrm{C}$ in $5 \% \mathrm{CO}_{2}$. After the infection, cells were overlaid with DMEM supplemented with 1.2\% Avicel (FMC, Philadelphia, PA, USA), 2\% HI-FBS, 1X nonessential amino acids (Gibco), and 1\% PEST. After 3 days, the overlays were removed, and cells were fixed by methanol (Fisher, Trinidad and Tobago) for $20 \mathrm{~min}$. The fixed cells were stained with 
staining buffer containing 2\% crystal violet (Sigma), 20\% methanol, and $0.1 \%$ ammonium oxalate (Sigma) solution for $1 \mathrm{~h}$ before washing in water.

The titer of CCHFV neutralizing antibodies in the serum of immunized mice was determined by microneutralization assay. The CCHFV strain Kosova Hoti (07v-EVA70) was obtained from the European Virus Archive. Sera from all mice in the Luc RVPs or the PBS control group were pooled, and sera from mice immunized with CCHFV Gn-Gc RVPs were divided into two groups (high or low anti-CCHFV Gn-Gc antibodies). Thereafter, the sera were heat-inactivated for $30 \mathrm{~min}$ at $56^{\circ} \mathrm{C}$. Serial 2-fold dilutions of sera were mixed with $200 \mathrm{CCHFV}$ Hoti viral particles and incubated at $37^{\circ} \mathrm{C}$ for $1 \mathrm{~h}$. Thereafter, $100 \mu \mathrm{L}$ of the serum-virus mix was added in duplicate to Vero cells on a 96 -well plate (20,000 cells/well). After $1 \mathrm{~h}$ incubation at $37^{\circ} \mathrm{C}$, the inoculum was removed, and cells were washed 3 times with DMEM supplemented with $2 \%$ FBS. Then, $100 \mu \mathrm{L}$ of DMEM supplemented with $2 \%$ FBS was added, and the cells were incubated for $24 \mathrm{~h}$ at $37^{\circ} \mathrm{C}$ and $5 \% \mathrm{CO}_{2}$. Cells were fixed with an ice-cold acetone-methanol mix (1:1) in $-20^{\circ} \mathrm{C}$ overnight and stained for CCHFV nucleoprotein for enumeration of fluorescent foci. Total numbers of infected cells in each well were counted and the result was expressed as percent neutralization compared to infection in wells with serum from the control group for each dilution.

\subsection{Statistics}

Statistical differences between the means of parametric data (weights) were determined using two-way ANOVA, followed by Bonferroni correction, while statistical differences between non-parametric data (absorbance values) were determined using MannWhitney test. GraphPad Prism 9 was used to perform all statistical analyses. The values are presented as mean \pm standard error of the mean.

\section{Conclusions}

In conclusion, this study has shed new light on ways to improve the $\mathrm{WNV}_{\mathrm{KUN}} \mathrm{RVPs}$ as a vaccine platform against $W N V$. Our results suggest that the $W N V_{K U N}$ RVPs have the capacity to transduce cells to express CCHFV Gn and Gc. The RVPs also enhanced the seroconversion and the production of neutralizing antibodies against WNV, which can increase the potential of the RVPs as a vaccine strategy against WNV.

Author Contributions: Conceptualization-W.M.; methodology-P.-T.-H.T., U.H., S.A. and O.L.; software-P.-T.-H.T.; formal analysis-P.-T.-H.T.; writing (original draft preparation)-P.-T.-H.T.; writing (review and editing) -P.-T.-H.T., M.J., S.A., A.M. and W.M.; visualization-P.-T.-H.T.; supervisionM.J. and W.M.; project administration-W.M.; and funding acquisition-M.J. All authors have read and agreed to the published version of the manuscript.

Funding: This research was funded by grants to M.J. from the Knowledge Foundation: Synergi 19 (20200063). The funding body played no role in the design of the study and collection, analysis, and interpretation of data, and in writing the manuscript.

Institutional Review Board Statement: The animal studies were conducted in accordance with ethics approval (4570-2019) by the Regional Animal Experimental Ethics Committee in Stockholm (North), Sweden.

Informed Consent Statement: Not applicable.

Data Availability Statement: The data presented in this study are available in this article. Remaining data supporting reported results are available from the corresponding authors upon reasonable requests.

Conflicts of Interest: The authors declare no conflict of interest. 


\section{References}

1. Lanciotti, R.S.; Ebel, G.D.; Deubelc, V.; Kerst, A.J.; Murric, S.; Meyerd, R.; Bowend, M.; Mc Kinneyd, N.; Morrill, W.E.; Crabtree, M.B.; et al. Complete Genome Sequences and Phylogenetic Analysis of West Nile Virus Strains Isolated from the United States, Europe, and the Middle East. Virology 2002, 298, 96-105. [CrossRef] [PubMed]

2. American CDC. Available online: https://www.cdc.gov/westnile/index.html (accessed on 24 June 2021).

3. Scherret, J.H.; Poidinger, M.; Mackenzie, J.S.; Broom, A.K.; Deubel, V.; Lipkin, W.I.; Briese, T.; Gould, E.A.; Hall, R.A. The relationships between West Nile and Kunjin viruses. Emerg. Infect. Dis. 2001, 7, 697-705. [CrossRef] [PubMed]

4. Hall, R.A.; Broom, A.K.; Smith, D.W.; Mackenzie, J.S. The Ecology and Epidemiology of Kunjin Virus. In Japanese Encephalitis and West Nile Viruses; Mackenzie, J.S., Barrett, A.D.T., Deubel, V., Eds.; Springer: Berlin/Heidelberg, Germany, $2002 ;$ pp. 253-269.

5. Kellman, E.M.; Offerdahl, D.K.; Melik, W.; Bloom, M.E. Viral Determinants of Virulence in Tick-Borne Flaviviruses. Viruses 2018, 10, 329. [CrossRef]

6. Barrows, N.J.; Campos, R.K.; Liao, K.-C.; Prasanth, K.R.; Soto-Acosta, R.; Yeh, S.-C.; Schott-Lerner, G.; Pompon, J.; Sessions, O.M.; Bradrick, S.S.; et al. Biochemistry and Molecular Biology of Flaviviruses. Chem. Rev. 2018, 118, 4448-4482. [CrossRef] [PubMed]

7. Zhang, X.; Xie, X.; Xia, H.; Zou, J.; Huang, L.; Popov, V.L.; Chen, X.; Shi, P.-Y. Zika Virus NS2A-Mediated Virion Assembly. mBio 2019, 10, e02375-19. [CrossRef] [PubMed]

8. Xie, X.; Zou, J.; Zhang, X.; Zhou, Y.; Routh, A.; Kang, C.; Popov, V.L.; Chen, X.; Wang, Q.-Y.; Dong, H.; et al. Dengue NS2A Protein Orchestrates Virus Assembly. Cell Host Microbe 2019, 26, 606-622.e8. [CrossRef] [PubMed]

9. Shi, P.-Y.; Tilgnera, M.; Lo, M. Construction and Characterization of Subgenomic Replicons of New York Strain of West Nile Virus. Virology 2002, 296, 219-233. [CrossRef]

10. Fayzulin, R.; Scholle, F.; Petrakova, O.; Frolov, I.; Mason, P.W. Evaluation of replicative capacity and genetic stability of West Nile virus replicons using highly efficient packaging cell lines. Virology 2006, 351, 196-209. [CrossRef]

11. Alcaraz-Estrada, S.L.; del Angel, R.; Padmanabhan, R. Construction of Self-Replicating Subgenomic Dengue Virus 4 (DENV4) Replicon. Program. Necrosis 2014, 1138, 131-150. [CrossRef]

12. Manokaran, G.; Sujatmoko; McPherson, K.G.; Simmons, C.P. Attenuation of a dengue virus replicon by codon deoptimization of nonstructural genes. Vaccine 2019, 37, 2857-2863. [CrossRef]

13. Khromykh, A.A.; Westaway, E.G. Subgenomic replicons of the flavivirus Kunjin: Construction and applications. J. Virol. 1997, 71, 1497-1505. [CrossRef] [PubMed]

14. Yamanaka, A.; Suzuki, R.; Konishi, E. Evaluation of single-round infectious, chimeric dengue type 1 virus as an antigen for dengue functional antibody assays. Vaccine 2014, 32, 4289-4295. [CrossRef] [PubMed]

15. Li, S.-H.; Li, X.-F.; Zhao, H.; Deng, Y.-Q.; Yu, X.-D.; Zhu, S.-Y.; Jiang, T.; Ye, Q.; Qin, E.-D.; Qin, C.-F. Development and characterization of the replicon system of Japanese encephalitis live vaccine virus SA14-14-2. Virol. J. 2013, 10, 64. [CrossRef] [PubMed]

16. Li, W.; Ma, L.; Guo, L.-P.; Wang, X.-L.; Zhang, J.-W.; Bu, Z.-G.; Hua, R.-H. West Nile virus infectious replicon particles generated using a packaging-restricted cell line is a safe reporter system. Sci. Rep. 2017, 7, 3286. [CrossRef]

17. Reynard, O.; Mokhonov, V.; Mokhonova, E.; Leung, J.; Page, A.; Mateo, M.; Pyankova, O.; Georges-Courbot, M.C.; Raoul, H.; Khromykh, A.A.; et al. Kunjin Virus Replicon-Based Vaccines Expressing Ebola Virus Glycoprotein GP Protect the Guinea Pig Against Lethal Ebola Virus Infection. J. Infect. Dis. 2011, 204 (Suppl. 3), S1060-S1065. [CrossRef]

18. Bente, D.A.; Forrester, N.L.; Watts, D.M.; McAuley, A.J.; Whitehouse, C.A.; Bray, M. Crimean-Congo hemorrhagic fever: History, epidemiology, pathogenesis, clinical syndrome and genetic diversity. Antivir. Res. 2013, 100, 159-189. [CrossRef] [PubMed]

19. WHO. Available online: https://www.who.int/health-topics/crimean-congo-haemorrhagic-fever/\#tab=tab_1 (accessed on 24 June 2021).

20. WHO. West Nile Virus. Available online: https://www.who.int/news-room/fact-sheets/detail/west-nile-virus (accessed on 24 June 2021)

21. Spengler, J.R.; Bergeron, É.; Spiropoulou, C.F. Crimean-Congo hemorrhagic fever and expansion from endemic regions. Curr. Opin. Virol. 2019, 34, 70-78. [CrossRef]

22. American CDC. Available online: https://www.cdc.gov/westnile/statsmaps/cumMapsData.html (accessed on 24 June 2021).

23. Shahhosseini, N.; Jafarbekloo, A.; Telmadarraiy, Z.; Chinikar, S.; Haeri, A.; Nowotny, N.; Groschup, M.H.; Fooks, A.R.; Faghihi, F. Co-circulation of Crimean-Congo Hemorrhagic Fever virus strains Asia 1 and 2 between the border of Iran and Pakistan. Heliyon 2017, 3, e00439. [CrossRef]

24. Tran, P.-T.-H.; Asghar, N.; Höglund, U.; Larsson, O.; Haag, L.; Mirazimi, A.; Johansson, M.; Melik, W. Development of a Multivalent Kunjin Virus Reporter Virus-Like Particle System Inducing Seroconversion for Ebola and West Nile Virus Proteins in Mice. Microorganisms 2020, 8, 1890. [CrossRef]

25. Zaayman, D.; Venter, M. West Nile Virus Neurologic Disease in Humans, South Africa, September 2008-May 2009. Emerg. Infect. Dis. 2012, 18, 2051-2054. [CrossRef]

26. Hall, R.A.; Nisbet, D.J.; Pham, K.B.; Pyke, A.; Smith, G.A.; Khromykh, A. DNA vaccine coding for the full-length infectious Kunjin virus RNA protects mice against the New York strain of West Nile virus. Proc. Natl. Acad. Sci. USA 2003, 100, 10460-10464. [CrossRef] [PubMed]

27. Paz, S.; Semenza, J.C. Environmental Drivers of West Nile Fever Epidemiology in Europe and Western Asia-A Review. Int. J. Environ. Res. Public Health 2013, 10, 3543-3562. [CrossRef] [PubMed] 
28. Kaiser, J.A.; Barrett, A.D. Twenty Years of Progress Toward West Nile Virus Vaccine Development. Viruses 2019, 11, 823. [CrossRef] [PubMed]

29. Ulbert, S. West Nile virus vaccines-Current situation and future directions. Hum. Vaccines Immunother. 2019, 15, 2337-2342. [CrossRef] [PubMed]

30. Rios, M.; Zhang, M.J.; Grinev, A.; Srinivasan, K.; Daniel, S.; Wood, O.; Hewlett, I.K.; Dayton, A.I. Monocytes-macrophages are a potential target in human infection with West Nile virus through blood transfusion. Transfusion 2006, 46, 659-667. [CrossRef]

31. García-Nicolás, O.; Lewandowska, M.; Ricklin, M.E.; Summerfield, A. Monocyte-Derived Dendritic Cells as Model to Evaluate Species Tropism of Mosquito-Borne Flaviviruses. Front. Cell. Infect. Microbiol. 2019, 9, 5. [CrossRef]

32. Rawle, D.J.; Setoh, Y.X.; Edmonds, J.H.; Khromykh, A.A. Comparison of attenuated and virulent West Nile virus strains in human monocyte-derived dendritic cells as a model of initial human infection. Virol. J. 2015, 12, 46. [CrossRef]

33. Ye, C.; Abraham, S.; Wu, H.; Shankar, P.; Manjunath, N. Silencing Early Viral Replication in Macrophages and Dendritic Cells Effectively Suppresses Flavivirus Encephalitis. PLoS ONE 2011, 6, e17889. [CrossRef]

34. Khan, M.S.A.; Nain, Z.; Syed, S.B.; Abdulla, F.; Moni, M.A.; Sheam, M.M.; Karim, M.M.; Adhikari, U.K. Computational formulation and immune dynamics of a multi-peptide vaccine candidate against Crimean-Congo hemorrhagic fever virus. Mol. Cell. Probes 2021, 55, 101693. [CrossRef]

35. Goedhals, D.; Paweska, J.T.; Burt, F.J. Long-lived CD8+ T cell responses following Crimean-Congo haemorrhagic fever virus infection. PLoS Neglected Trop. Dis. 2017, 11, e0006149. [CrossRef]

36. Shepherd, A.J.; Swanepoel, R.; Leman, P.A. Antibody Response in Crimean-Congo Hemorrhagic Fever. Clin. Infect. Dis. 1989, 11, S801-S806. [CrossRef] [PubMed]

37. Bertolotti-Ciarlet, A.; Smith, J.; Strecker, K.; Paragas, J.; Altamura, L.A.; McFalls, J.M.; Frias-Stäheli, N.; Garcia-Sastre, A.; Schmaljohn, C.S.; Doms, R.W. Cellular Localization and Antigenic Characterization of Crimean-Congo Hemorrhagic Fever Virus Glycoproteins. J. Virol. 2005, 79, 6152-6161. [CrossRef] [PubMed]

38. Tran, P.-T.-H.; Asghar, N.; Johansson, M.; Melik, W. Roles of the Endogenous Lunapark Protein during Flavivirus Replication. Viruses 2021, 13, 1198. [CrossRef] [PubMed] 